University of Nebraska - Lincoln

DigitalCommons@University of Nebraska - Lincoln

USDA National Wildlife Research Center - Staff Publications
U.S. Department of Agriculture: Animal and Plant Health Inspection Service

2010

\title{
Wildlife Conservation Sunflower Plots and Croplands as Fall Habitat for Migratory Birds
}

\author{
Heath M. Hagy \\ North Dakota State University, hhagy@cfr.msstate.edu \\ George M. Linz \\ USDA/APHIS/WS National Wildlife Research Center, george.m.linz@aphis.usda.gov \\ William J. Bleier \\ North Dakota State University, William.Bleier@ndsu.nodak
}

Follow this and additional works at: https://digitalcommons.unl.edu/icwdm_usdanwrc

Hagy, Heath M.; Linz, George M.; and Bleier, William J., "Wildlife Conservation Sunflower Plots and Croplands as Fall Habitat for Migratory Birds" (2010). USDA National Wildlife Research Center - Staff Publications. 1273.

https://digitalcommons.unl.edu/icwdm_usdanwrc/1273

This Article is brought to you for free and open access by the U.S. Department of Agriculture: Animal and Plant Health Inspection Service at DigitalCommons@University of Nebraska - Lincoln. It has been accepted for inclusion in USDA National Wildlife Research Center - Staff Publications by an authorized administrator of DigitalCommons@University of Nebraska - Lincoln. 


\title{
Wildlife Conservation Sunflower Plots and Croplands as Fall Habitat for Migratory Birds
}

\author{
HEATH M. HAGY ${ }^{1}$ \\ Department of Biological Sciences, North Dakota State University, Stevens Hall, Fargo 58105 \\ GEORGE M. LINZ \\ United States Department of Agriculture, Wildlife Services, National Wildlife Research Center, Bismarck, \\ North Dakota 58501 \\ AND \\ WILLIAM J. BLEIER \\ Department of Biological Sciences, North Dakota State University, Stevens Hall, Fargo 58105
}

\begin{abstract}
Agricultural fields are often overlooked as post-breeding and migratory bird habitat, even though many species use row-crop fields in the northern Great Plains. We monitored bird use, crop and non-crop vegetation characteristics and abundance, and land use around $(\leq 2.4 \mathrm{~km}) 35$ 8-ha Wildlife Conservation Sunflower Plots (WCSP) and one commercial sunflower and non-sunflower row-crop field, paired with each WCSP, in fall 2004 and 2005. We excluded four species of blackbirds from our analysis that commonly form large foraging flocks and ubiquitously use agricultural fields. A diverse community of migratory birds used WCSP and commercial sunflower compared to other non-sunflower crops in North Dakota. Both WCSP $($ mean $=24.4$ birds $/$ ha, $\mathrm{SE}=2.7$ ) and commercial sunflower $($ mean $=12.7$ birds $/$ ha, $\mathrm{SE}=1.7)$ harbored greater densities of birds $(\mathrm{P}<0.01)$ than did other non-sunflower row crops $($ mean $=7.2$ birds $/$ ha, $\mathrm{sE}=1.1)$. Migratory birds were more strongly associated with vegetation within fields, such as crop density $(+)$, non-crop plant abundance $(+)$ and crop height $(+)$, than surrounding land uses $(0-2.4 \mathrm{~km}$ from WCSP). We recommend management practices to maximize WCSP for fall bird habitat and discuss economic considerations for WCSP implementation as a wildlife habitat / blackbird damage management system.
\end{abstract}

\section{INTRODUCTION}

Agricultural crops provide habitat for migratory birds in the northern Great Plains (NGP) of North America during and before migration periods. Extensive cultivation of crops has fragmented $>50 \%$ of prairies in the NGP and likely influenced bird communities in the region (Peterjohn, 2003; Lubowski et al., 2006). Few studies have examined migratory bird use of croplands in North America, especially during non-breeding periods, even though croplands represent the third-largest land use (19.5\%) in the United States (Lubowski et al., 2006).

Birds migrating during fall likely select staging habitat hierarchically based on foraging and energy demands of migration, landscape composition and weather (Johnson, 1980; Kolasa, 1989; Kotliar and Wiens, 1990; Bergin, 1992; Moore and Simons, 1992; Moore and Aborn, 2000). Croplands may be selected based on patch quality detectable from proximate cues or composition of habitat complexes (Best et al., 1990, 1998; Flather and Sauer, 1996;

\footnotetext{
${ }^{1}$ Corresponding author present address: Department of Wildlife and Fisheries, Mississippi State University, Box 9690, Mississippi State 39762; e-mail: hhagy@cfr.msstate.edu; Telephone: (662) 3254739
} 
Koford and Best, 1996; Schaaf, 2003; Galle, 2005; Pearse, 2007). Within selected landscapes, land use diversity surrounding roost and loafing sites and vegetative characteristics within fields likely influence field selection and subsequent use (Stone and Danner, 1980; Best et al., 2001; Cunningham and Johnson, 2006). Some croplands within the NGP may provide disproportionately important habitat given crop characteristics, farming practices, time of harvest relative to other crops and associated food resources.

Birds use sunflower and other crops during migration through the NGP (Best et al., 1998; Murphy, 2003; Hagy et al., 2007), but we know little about landscape-scale or within-field variables influencing use (Moore et al., 1995; Peterjohn, 2003). Sunflower is used extensively by migrating birds, especially after mid- to late-summer harvest and tilling of other row crops reduce vegetation and vertical structure of croplands in the NGP (Schaaf, 2003; Linz et al., 2003). In late summer and fall, an energy-rich and structurally diverse crop (Schaff, 2003) such as sunflower may be easily recognizable as a source of cover and food for a variety of birds (Charlet et al., 1997; Linz et al., 2004; Hagy et al., 2007).

Blackbirds [red-winged blackbirds (Agelaius phoeniceus), Brewer's blackbird (Euphagus cyanocephalus), common grackles (Quiscalus quiscula) and yellow-headed blackbirds (Xanthocephalus xanthocephalus)] extensively use sunflower fields and subsequently cause economically important damage by feeding on ripening sunflower seeds (Otis and Kilburn, 1988; Peer et al., 2003; Hagy et al., 2008). Traditional harassment and damage prevention methods are time-intensive, expensive and negatively affect other wildlife and bird species (Kleingartner, 2003; Linz et al., 2003). Furthermore, population declines of several blackbird species (Brewer's blackbird, red-winged blackbird and common grackle) since 1966 and negative public sentiment associated with lethal population control incentivize non-lethal pest management practices (Kleingartner, 2003; Sauer et al., 2008). Blackbird depredation of commercial sunflower can be mediated by strategically planting small sunflower lure plots to concentrate blackbird foraging flocks away from commercial sunflower fields (Cummings et al., 1987; Hagy et al., 2008). Additionally, lure plots may provide stopover habitat for other migratory bird species and wildlife and reduce disturbance of non-blackbird species using commercial sunflower fields.

Farmers contracted with the United States Department of Agricultural/Animal and Plant Health Inspection Service's division of Wildlife Services to plant 8 ha plots of oilseed sunflower in $2004(\mathrm{n}=14)$ and $2005(\mathrm{n}=21)$, called Wildlife Conservation Sunflower Plots (WCSP). WCSP were planted to lure blackbirds away from commercial sunflower fields and provide habitat for other migratory birds and wildlife (Cummings et al., 1987). Cost-share opportunities to plant WCSP were awarded to farmers based on historical county-wide damage estimates, history of damage on site and presence of cattail-dominated wetlands that blackbirds typically use as night roosts in proposed county sections. Individual farmers who were accepted in the WCSP program determined placement within the approved county section and planted WCSP. Hagy et al. (2008) reported guidelines for maximizing WCSP placement to reduce blackbird damage to sunflower, but did not describe use of plots or surrounding croplands by other bird species, which was a primary objective of WCSP program and Wildlife Services. Cummings et al. (1987) evaluated a similar program in the early 1980s and found that bird use of decoy plots was largely influenced by the surrounding landscape, but this included only blackbird species.

Here, we (1) compare bird use [excluding blackbirds which was described by Hagy et al. (2008) ], among WCSP, commercial sunflower, and other non-sunflower row-crop fields, (2) describe the within-field and surrounding landscape characteristics of WCSP that were related to bird use and (3) develop guidelines for future placement and management of 
WCSP in the NGP so that agricultural producers, conservation planners and habitat managers can collectively improve migratory bird habitat while retaining productive agricultural practices.

\section{Methods \\ STUDY AREA}

We surveyed 35 sites in late summer and fall 2004 and 2005 in the Prairie Pothole Region (PPR) of east-central North Dakota. Each site included one WCSP, one nearby $[\leq 2.4 \mathrm{~km}$ from WCSP] commercial sunflower field, and one nearby non-sunflower row-crop field. Sites were located at least $4.5 \mathrm{~km}$ apart and the study area encompassed $\sim 60,000 \mathrm{~km}^{2}$. Rowcrop agriculture (canola, flax, lentils, oats, soybeans, sunflower and wheat), grasslands, shelterbelts and wetlands are the most common pre-migratory and stopover habitats available to birds in this region (Stewart, 1975; USDA, 2008). Approximately 400,000 ha of sunflower are planted annually in North Dakota (USDA, 2008), more than any other state. Sunflower is an important commodity in this region due to local processing and refining infrastructure.

\section{FIELD METHODS}

We surveyed 14 WCSP and paired fields from 24 Aug.-19 Oct. 2004 three times at 2 wk intervals and 21 WCSP and paired fields from 10 Aug.-28 Oct. 2005 two times at 4 wk intervals. We conducted fixed radius $(50 \mathrm{~m})$ bird point counts and vegetation measurements at one site (1 WCSP $[$ mean $=8.0$ ha, $\mathrm{SE}=0.1]$ and one nearby, randomly selected commercial sunflower [mean $=33.7 \mathrm{ha}, \mathrm{sE}=2.9]$ and non-sunflower row-crop field $[$ mean $=26.9 \mathrm{ha}, \mathrm{SE}=2.3])$ per day. We selected commercial fields where bird disturbance and blackbird harassment effort from producers appeared to be minimal. We used aerial photographs and ground surveys to measure field dimensions and divided all fields into square 1-ha units prior to bird and vegetation surveys. We overlaid a square 1-ha grid pattern over the aerial imagery of each field and selected locations for the bird point counts and subsequent vegetation surveys. As field sizes varied (8-90 ha), we randomly selected $15 \%$ of the total ha in each field, during each survey round, for bird point counts with a minimum of 2 point counts per field. Given large field sizes, $15 \%$ was typically the maximum area one observer could survey in one day. We chose a conservative count distance of $50 \mathrm{~m}$ as habitat structure and consequently species detectability varied across field-types (Cunningham and Johnson, 2006) and long-distance point counts can result in considerable error in abundance estimates (Efford and Dawson, 2009). We did not count birds in adjacent ha to reduce the chance of double-counting and used the same commercial sunflower and nonsunflower row-crop fields during each survey round. We randomly selected the order of fields for bird surveys each time we visited a site.

We used distance sampling methods where one observer estimated and recorded distance to each bird seen flying or perching 0-50 m from the center of each ha beginning $30 \mathrm{~min}$ after sunrise. We waited $3 \mathrm{~min}$ after arriving at the center of each survey ha and then conducted an 8 min visual bird survey. A $2 \mathrm{~m}$ step-ladder was used in tall crops (e.g., sunflower and corn) to aid bird observations. One observer conducted all counts in 2004, and two observers conducted all counts in 2005. In 2005, one observer completed all surveys for a given site during a given survey round, individually and observers periodically practiced bird identification and distance estimation together prior to point counts to increase accuracy and precision of data collection. We did not conduct surveys in dense fog, when raining or snowing, or when winds exceeded $20 \mathrm{kph}$ (Ribic et al., 2009a). 
Bird surveys were usually completed in all fields prior to 1000 (CDT). After completion, we returned to each previous bird-survey ha and measured vegetation characteristics in two, randomly selected $1-\mathrm{m}^{2}$ subplots per bird-survey ha. Measurements included crop density (number of crop plants), vertical obstruction (measured with a $15 \mathrm{~cm} \times 2 \mathrm{~m}$ density board placed in the middle of the subplot), row width, percent canopy cover (measured by placing a spherical densitometer on the ground in the center of the subplot and counting unobscured squares), weed density (number of non-crop plants), waste seed abundance (seeds were vacuumed from the entire subplot using a portable vacuum), maximum plant height and identification to family of all non-crop plant species present in the subplots. Waste seed abundance was determined by washing all materials through a large $(4.5 \mathrm{~mm})$ and small $(0.3 \mathrm{~mm})$ sieve, drying seeds and detritus, and manually removing, counting and weighing seeds (natural and from the crop). Data from two subplots was averaged to generate one set of vegetation metrics per survey ha before analysis. Sites were surveyed in the same order within each round of surveys as the large size of the study area made randomizing site visitation order logistically impractical.

In late summer 2004 and 2005, we visually surveyed and delineated all major land-uses within $2.4 \mathrm{~km}$ of each WCSP (Cummings et al., 1987; Cunningham and Johnson, 2006). We obtained aerial photography from the USDA-NRCS Geospatial Data Gateway (USDA, 2005) and manually overlaid land use data within $2.4 \mathrm{~km}$ of each WCSP in ArcMap (ESRI, 2004).

Statistical analysis. - We counted blackbirds, but omitted them from analysis as they form large foraging flocks and were periodically abundant enough to overwhelm analysis compared to other species (Otis and Kilburn, 1988; Schaff, 2003). Hagy et al. (2008) reported on blackbird use of WCSP and surrounding agricultural habitats. Other studies have similarly omitted them from analysis due to their ubiquitous habitat use (Ribic et al., 2009a) and behavior (Lokemoen and Beiser, 1997).

We used program DISTANCE (ver. 5.0; Thomas et al., 2005) to estimate bird densities using crop- and species-specific detection functions adjusting observations for detectabilities. We pooled observations among survey rounds, but not years or field types to estimate detection functions. We used the multi-covariate distance sampling engine in Program DISTANCE and detection functions for half-normal and hazard-rate key functions with cosine, simple polynomial and hermite polynomial series expansions with stratification at the site level (Marques et al., 2007). Different combinations of the above key functions and series expansions allow variable fitting of the distance estimation curve, thus improving accuracy of density estimates for each data set of bird observations due to different detectabilities. We constructed all models with and without the covariates of plant height and vertical cover, which were not strongly correlated. We selected the best models based on Chi-square model fit statistics, Akaike's information criterion (Akaike, 1974), shape and structure of the detection probability and probability plots, and biological plausibility of the density estimates (Buckland et al., 2001; Burnham and Anderson, 2002). We were not able to reliably estimate species-specific densities for each site due to few or variable detections. We grouped data into even intervals and left-truncated at $3 \mathrm{~m}$ for improved model fit after examining distance histograms to detect any rounding of distances by observers (Buckland et al., 2001).

We generated density estimates of all species combined ("all birds") using global and crop-specific detection functions and post stratification for all birds present in WCSP, commercial sunflower, and non-sunflower row-crop fields (Alldredge et al., 2007). Additionally, we estimated densities of species with at least 85 detections using speciesspecific detection functions. We used a global detection function (generated by pooling 
observations among all species) to generate species-specific density estimates for species with fewer than 85 detections (Buckland et al., 2001, p. 240).

We used an information theoretic approach to model all bird density as a function of habitat variables and constructed models using 22 habitat variables which included a prioriselected within-field vegetation metrics and surrounding land-use variables using linear mixed models (Proc MIXED in SAS, SAS Institute Inc., 2005). Models consisted of 2-5 independent variables based on our sample size $(n=35)$ compared to the dependent variable, all bird density.

We examined bird densities and all independent variables (Table 1) for correlations and deviation from normality using Microsoft Excel Pop Tools and JMP (SAS Institute Inc., 2005). Non-normal data were transformed using either $\ln (\mathrm{x}+1)$ or $\operatorname{sqrt}(\mathrm{x})$ transformations as appropriate to achieve a normal distribution and homogeneity of variance (Zar, 1999). We designated year as a random effect and survey round as the repeated measure. We used model selection based on Akaike's second order Information Criterion $\left(\mathrm{AIC}_{\mathrm{c}}\right)$ to evaluate all models (Akaike, 1974; Burnham and Anderson, 2002).

We formed 2 sets of models for WCSP, an a priori and post hoc model set. We constructed $a$ priori models based on previously published bird-habitat relationships and knowledge of bird ecology in agricultural habitats before completing data collection and analysis (Best $e t$ al., 1990, 1998, 2001; Crozier and Niemi, 2003). Post hoc models were formed because little published information exists on fall bird use of agricultural crop fields and we desired to use our experience gained while working in the study system to elucidate possible relationships between birds and landscape factors. Furthermore, in exploratory studies where little published information exists describing the study systems, it may be unreasonable to assume that researchers can formulate all possible and biologically plausible models before data collection (Hagy et al., 2008; Uyehara et al., 2008). Therefore, we formed post hoc models using SAS Proc MIXED to regress each independent variable (Table 1) singularly with each response variable and the random effect of year (null model), and ranked them from lowest AICc value (the best performing independent variables) to highest (the worst performing independent variable; Cunningham and Johnson, 2006; Hagy et al., 2008; Uyehara et al., 2008). We used these $\mathrm{AIC}_{\mathrm{c}}$ scores and a posteriori knowledge of the ecological system to construct only biologically plausible post hoc models (Anderson et al., 2000; Johnson and Omland, 2004). The formation of only biologically plausible models has been recommended over running all possible models to avoid spurious findings (Johnson and Omland, 2004). We labeled all models by the variables they contained (intrinsic WCSP metrics, extrinsic WCSP metrics and mixed metrics) to evaluate the relative influence of intrinsic (habitat metrics measured from within each field such as crop density) versus extrinsic [habitat characteristics measured outside of each field such as nearby $(\leq 2.4 \mathrm{~km})$ wetland ha] characteristics on bird density (Moore and Simons, 1992; Cunningham and Johnson, 2006). All final models were evaluated using SAS (Proc MIXED) and maximum likelihood estimation as we varied the fixed effects throughout the final models in each set (Littell et al., 1996; Riffell et al., 2006).

We assessed relative variable importance using only models in the $90 \%$ confidence set whose weights collectively summed to $\geq 0.90$, since several models were supported (Burnham and Anderson, 2002; Riffell et al., 2006). We ranked all competing models by $\Delta \mathrm{AIC}_{\mathrm{c}}$, calculated model weights and model averaged parameter estimates, and estimated relative variable importance with variances from the $90 \%$ confidence set (Burnham and Anderson, 2002). Interpretation of independent variable importance was based on model averaged parameter estimates and the frequency of inclusion within the $90 \%$ confidence set. 
Table 1.-Bird detections (N) and density estimates (birds/ha; D) with corresponding standard errors (SE) and variation in density attributable to detection probability $\left(\% \hat{\sigma}^{2}\right)$ in WCSP, commercial sunflower and non-sunflower row crops generated by fitting global detection probabilities in DISTANCE to point-count observations

\begin{tabular}{|c|c|c|c|c|c|c|c|c|c|c|c|c|}
\hline \multirow[b]{2}{*}{ Species } & \multicolumn{4}{|c|}{ WCSP } & \multicolumn{4}{|c|}{ SUNFLOWER } & \multicolumn{4}{|c|}{ CROP } \\
\hline & $\mathrm{N}$ & D & $\mathrm{SE}$ & $\% \hat{\sigma}^{2}$ & $\mathrm{~N}$ & D & SE & $\% \hat{\sigma}^{2}$ & $\mathrm{~N}$ & $\mathrm{D}$ & $\mathrm{SE}$ & $\% \hat{\sigma}^{2}$ \\
\hline American Goldfir & & & & & & & & & & & & \\
\hline Carduelis tristis & 163 & 3.91 & 0.97 & 9.3 & 312 & 2.63 & 0.41 & 2 & 4 & 0.05 & 0.03 & 0.4 \\
\hline
\end{tabular}

American Pipit

Anthus rubescens

American Robin

Turdus migratorius

$7 \quad 0.16-0.14$

Barn Swallow

Hirundo rustica

$53 \quad 1.27 \quad 0.42$

$\begin{array}{lllll}5.2 & 123 & 1.03 & 0.28 & 0.6\end{array}$

$\begin{array}{llll}197 & 2.64 & 0.85 & 1.3\end{array}$

Blue Jay

Cyanocitta cristata

Bobolink

Dolichonyx oryzivorus

$\begin{array}{lllllll}16 & 0.38 & 0.23 & 1.6 & 31 & 0.26 & 0.15\end{array}$

Brown Thrasher

Toxostoma rufrum

Cedar Waxwing

Bombycilla cedrorum

Chipping Sparrow

Spizella passerina

Clay-colored Sparrow

Spizella pallida

Common Yellowthroat Geothlypis trichas

$\begin{array}{lll}3 & 0.72 & 0.41\end{array}$

0.48

0.2

3.

$\begin{array}{lll}35 & 0.29 & 0.19\end{array}$

0.1

$\begin{array}{llll}3 & 0.04 & 0.03 & 0.2\end{array}$

$\begin{array}{llllllllllll}144 & 3.45 & 0.7 & 13.9 & 226 & 1.9 & 0.31 & 1.7 & 10 & 0.13 & 0.05 & 0.7\end{array}$

Cooper's Hawk

$$
\text { Accipiter cooperii }
$$

Dark-eyed Junco

Junco hyemalis

Dickcissel

Spiza americana

Eastern Kingbird Tyrannus tyrannus

$\begin{array}{lll}3 & 0.07 & 0.07\end{array}$

$\begin{array}{llllllll}9 & 0.21 & 0.12 & 1.7 & 4 & 0.03 & 0.02 & 0.1\end{array}$

$\begin{array}{llllllll}3 & 0.72 & 0.53 & 1 & 1 & 0.01 & 0.01 & 0\end{array}$

$\begin{array}{llllllll}16 & 0.38 & 0.29 & 1 & 24 & 0.2 & 0.13 & 0.1\end{array}$

Evening Grosbeak Coccothraustes vespertinus

$\begin{array}{lll}1 & 0.02 & 0.02\end{array}$

Field Sparrow

Spizella pusilla

$\begin{array}{llllll}0.1 & 1 & 2 & 0.01 & 0.01 & 0\end{array}$

$\begin{array}{llll}5 & 0.06 & 0.05 & 0.2\end{array}$ 
TABLE 1.-Continued

\begin{tabular}{|c|c|c|c|c|c|c|c|c|c|c|c|c|}
\hline \multirow[b]{2}{*}{ Species } & \multicolumn{4}{|c|}{ WCSP } & \multicolumn{4}{|c|}{ SUNFLOWER } & \multicolumn{4}{|c|}{ CROP } \\
\hline & $\mathrm{N}$ & $\mathrm{D}$ & $\mathrm{SE}$ & $\% \hat{\sigma}^{2}$ & $\mathrm{~N}$ & $\mathrm{D}$ & $\mathrm{SE}$ & $\% \hat{\sigma}^{2}$ & $\mathrm{~N}$ & $\mathrm{D}$ & $\mathrm{SE}$ & $\% \hat{\sigma}^{2}$ \\
\hline
\end{tabular}

Fox Sparrow

Passerella iliaca

Franklin's Gull

Larus pipixcan

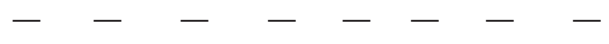

$\begin{array}{lll}1 & 0.1 & 0.01\end{array}$

0.1

Gray Partridge

Perdix perdix

$\begin{array}{lllllll}1 & 0.02 & 0.02 & 0.6 & - & - & -\end{array}$

Harris's Sparrow

Zonotrichia querula

$\begin{array}{llllllll}14 & 0.33 & 0.25 & 1 & 4 & 0.03 & 0.02\end{array}$

Horned Lark

\section{Eremophila alpestris}

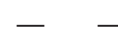

House Finch

Carpodacus mexicanus

House Sparrow

Passer domesticus

Killdeer

Charadrius vociferus

$10.02 \quad 0.02$

$0.6--\quad-$

$\begin{array}{llll}10 & 0.13 & 0.08 & 0.4\end{array}$

Lapland Longspur

Calcarius lapponicus

- - - - - - - - -

$12 \quad 0.16 \quad 0.13$

0.2

Lark Sparrow

Chondestes grammacus

Melospiza lincolnii

$\begin{array}{llllllllllll}8 & 0.19 & 0.1 & 2 & 19 & 0.16 & 0.08 & 0.2 & 5 & 0.06 & 0.03 & 0.5\end{array}$

Marsh Wren

Cistothorus palustris

$\begin{array}{lll}3 & 0.07 & 0.05\end{array}$

Mourning Dove

Zenaida macroura

$\begin{array}{llllllllllll}97 & 2.33 & 1.09 & 2.6 & 88 & 0.74 & 0.11 & 2 & 8 & 0.1 & 0.03 & 1.1\end{array}$

Northern Flicker

Colaptes auratus

Northern Harrier

Circus cyaneus

Northern Waterthrush

Seiurus noveboracensis

Orchard Oriole

Icterus spurius

Palm Warbler

Dendroica palmarum

Ring-necked Pheasant

Phasianus colchicus

$\begin{array}{lllllll}9 & 0.21 & 0.21 & 0.6 & 3 & 0.02 & 0.01\end{array}$

0.1 
TABle 1.-Continued

\begin{tabular}{|c|c|c|c|c|c|c|c|c|c|c|c|c|}
\hline \multirow[b]{2}{*}{ Species } & \multicolumn{4}{|c|}{ WCSP } & \multicolumn{4}{|c|}{ SUNFLOWER } & \multicolumn{4}{|c|}{ CROP } \\
\hline & $\mathrm{N}$ & $\mathrm{D}$ & SE & $\% \hat{\sigma}^{2}$ & $\mathrm{~N}$ & $\mathrm{D}$ & SE & $\% \hat{\sigma}^{2}$ & $\mathrm{~N}$ & $\mathrm{D}$ & SE & $\% \hat{\sigma}^{2}$ \\
\hline
\end{tabular}

Ruby-throated

Hummingbird

Archilochus colubris

$\begin{array}{llllllll}1 & 0.02 & 0.02 & 0.6 & 1 & 0.01 & 0.01 & 0\end{array}$

$\begin{array}{llll}1 & 0.01 & 0.01 & 0.1\end{array}$

Savannah Sparrow

Passerculus

$\begin{array}{lllllllllllll}\text { sandwichensis } & 143 & 3.4 & 0.83 & 9.8 & 127 & 1.07 & 0.2 & 1.3 & 33 & 0.44 & 0.11 & 2\end{array}$

Sharp-tailed Grouse

Tympanuchus

phasianellus

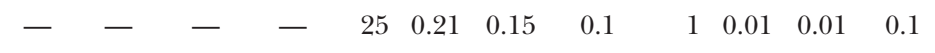

Song Sparrow

Melospiza melodia

$\begin{array}{lllllllllllll}27 & 0.64 & 0.32 & 2.3 & 14 & 0.11 & 0.06 & 0.1 & 3 & 0.04 & 0.04 & 0.1\end{array}$

Swamp Sparrow

Melospiza geogiana

$\begin{array}{llllllllllll}4 & 0.09 & 0.09 & 0.6 & 5 & 0.04 & 0.03 & 0.1 & - & - & - & -\end{array}$

Tennessee Warbler

Vermivora peregrina

$\begin{array}{lllllll}1 & 0.02 & 0.02 & 0.6 & - & - & -\end{array}$

Tree Swallow

Tachycineta bicolor

$31 \quad 0.74 \quad 0.34$

$\begin{array}{llll}2.7 & 49 & 0.41 & 0.17\end{array}$

0.3

$\begin{array}{llll}74 & 0.99 & 0.35 & 1\end{array}$

Vesper Sparrow

Pooecetes gramineus

$\begin{array}{llllllllllll}3 & 0.07 & 0.05 & 1 & 38 & 0.32 & 0.16 & 0.2 & 1 & 0.01 & 0.01 & 0.1\end{array}$

Western Meadowlark

Sturnella neglecta

White-crowned Sparrow

Zonotrichia leucophrys

$\begin{array}{lllllllllllllll}6 & 0.14 & 0.1 & 1 & 1 & 0.01 & 0.01 & 0 & - & - & - & -\end{array}$

White-throated Sparrow

Zonotrichia albicollis

$\begin{array}{llllllllllll}6 & 0.14 & 0.1 & 1.1 & 14 & 0.11 & 0.09 & 0.1 & 54 & 0.72 & 0.58 & 0.2\end{array}$

Yellow Warbler

\section{Dendroica petechia}

Yellow-bellied Flycatcher

Empidonax flaviventris

$\begin{array}{lllllll}1 & 0.02 & 0.02 & 0.6 & 12 & 0.1 & 0.05\end{array}$

$0.2-\quad-\quad-$

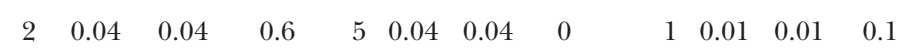

Yellow-rumped Warbler

Dendroica coronata

Unidentified Birds

$\begin{array}{llll}0.6 & 51 & 0.43 & 0.19\end{array}$

218

95

Effective Detection

Radius

$27.9 \pm 1.06$

$31.2 \pm 0.03$

$26.4 \pm 0.04$

Using bird densities generated by stratification by site in DISTANCE, we compared all bird densities across all field types using repeated measures analysis of variance (ANOVA). We assigned field type as the treatment effect, survey round as the repeated measure, and year as a random effect in Proc MIXED (SAS Institute Inc., 2005). We used post-hoc Tukey 


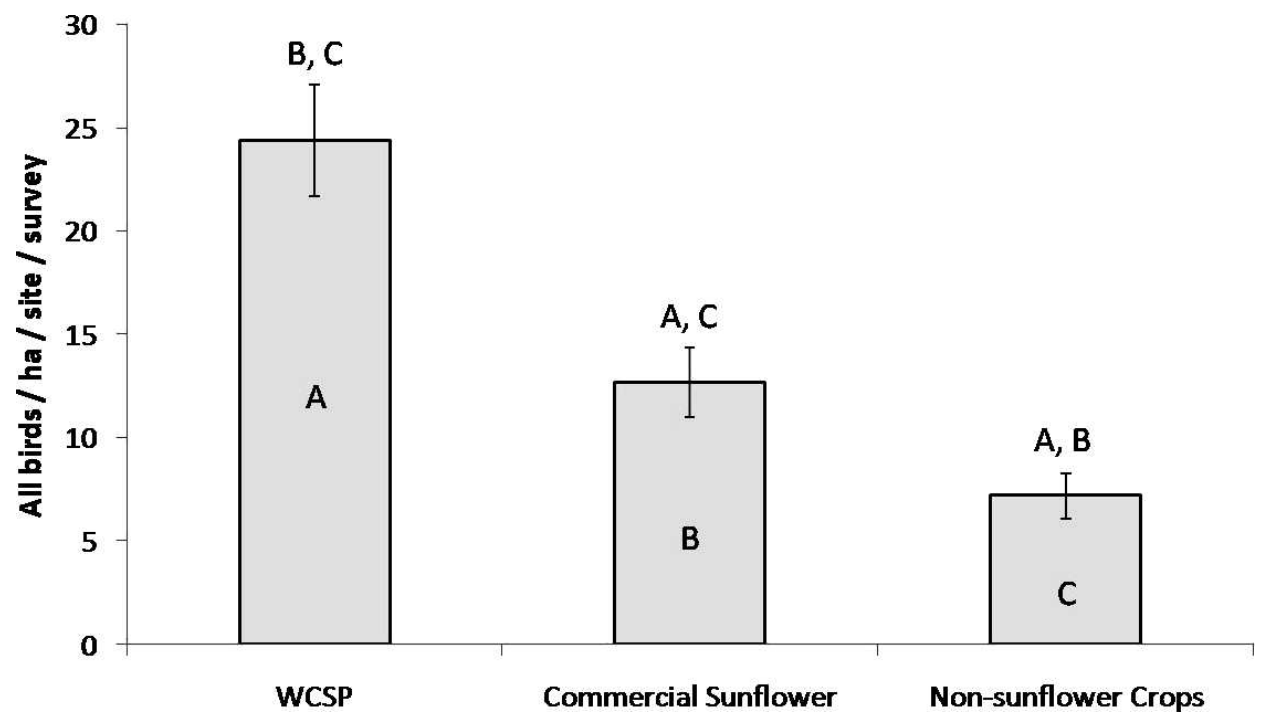

FIG 1. Mean all bird abundance (2004-2005) in WCSP, commercial sunflower and other nonsunflower row crops with associated standard errors.-Letters A, B and C represent $\alpha=0.05$ differences (Tukey's post hoc pair-wise comparisons among habitat types)

multiple comparisons $(\alpha=0.05)$ to test for differences between bird densities in WCSP, commercial sunflower, and non-sunflower row-crop fields (Zar, 1999). We used bird densities and non-crop plant abundance to calculate Simpson's Diversity Index for WCSP, commercial sunflower and non-sunflower row-crop fields (McCune and Grace, 2002). We similarly used a mixed model ANOVA to compare abundance of non-crop plants and Simpson's diversity among field types. We chose Simpson's diversity index because of concerns with other diversity indices expressed in Whittaker (1972).

\section{RESULTS}

Bird abundance.-We observed 34 bird species in WCSP (mean $=4.2$ species/ha), 37 in commercial sunflower $($ mean $=1.1$ species $/$ ha) and 24 in commercial non-sunflower rowcrops $($ mean $=0.8$ species $/$ ha; Table 1$)$. Birds occurred at greater densities $(\mathrm{P}<0.01)$ but similar diversities in WCSP (mean $=24.4$ birds $/$ ha, $\mathrm{SE}=2.7 ;$ Div $=0.88$ ), followed by commercial sunflower $($ mean $=12.7$ birds $/$ ha, $\mathrm{SE}=1.7 ;$ Div $=0.88)$ and non-sunflower rowcrops $($ mean $=7.2$ birds $/$ ha, $\mathrm{sE}=1.1 ;$ Div $=0.80 ;$ Fig. 1$)$. We recorded sufficient detections of individuals $(n \geq 85)$ to generate species-specific detection probabilities and densities for four species in WCSP, five species in commercial sunflower and two species in other nonsunflower row-crops (Table 2).

Influence of habitat variables. - The mean abundance of non-crop plants was similar $(\mathrm{P}=$ 0.71 ) among WCSP (mean $=53.9$ forbs $/ \mathrm{m}^{2}$, $\mathrm{SE}=11.9$ ), commercial sunflower (mean = 41.8 forbs $\left./ \mathrm{m}^{2}, \mathrm{SE}=10.7\right)$ and other non-sunflower row-crops $\left(\right.$ mean $=54.6$ forbs $/ \mathrm{m}^{2}, \mathrm{sE}=$ 12.7). Most non-crop plants were in the Poaceae family in all three field types $(61 \%$ in WCSP, $57 \%$ in commercial sunflower, and $87 \%$ in non-sunflower row-crop fields); however, Simpson's diversity index for non-crop plant abundance varied $(\mathrm{P}<0.01)$ among WCSP $($ Div $=0.96)$, commercial sunflower $($ Div $=0.87)$ and non-sunflower row crops $($ Div $=0.77)$. 


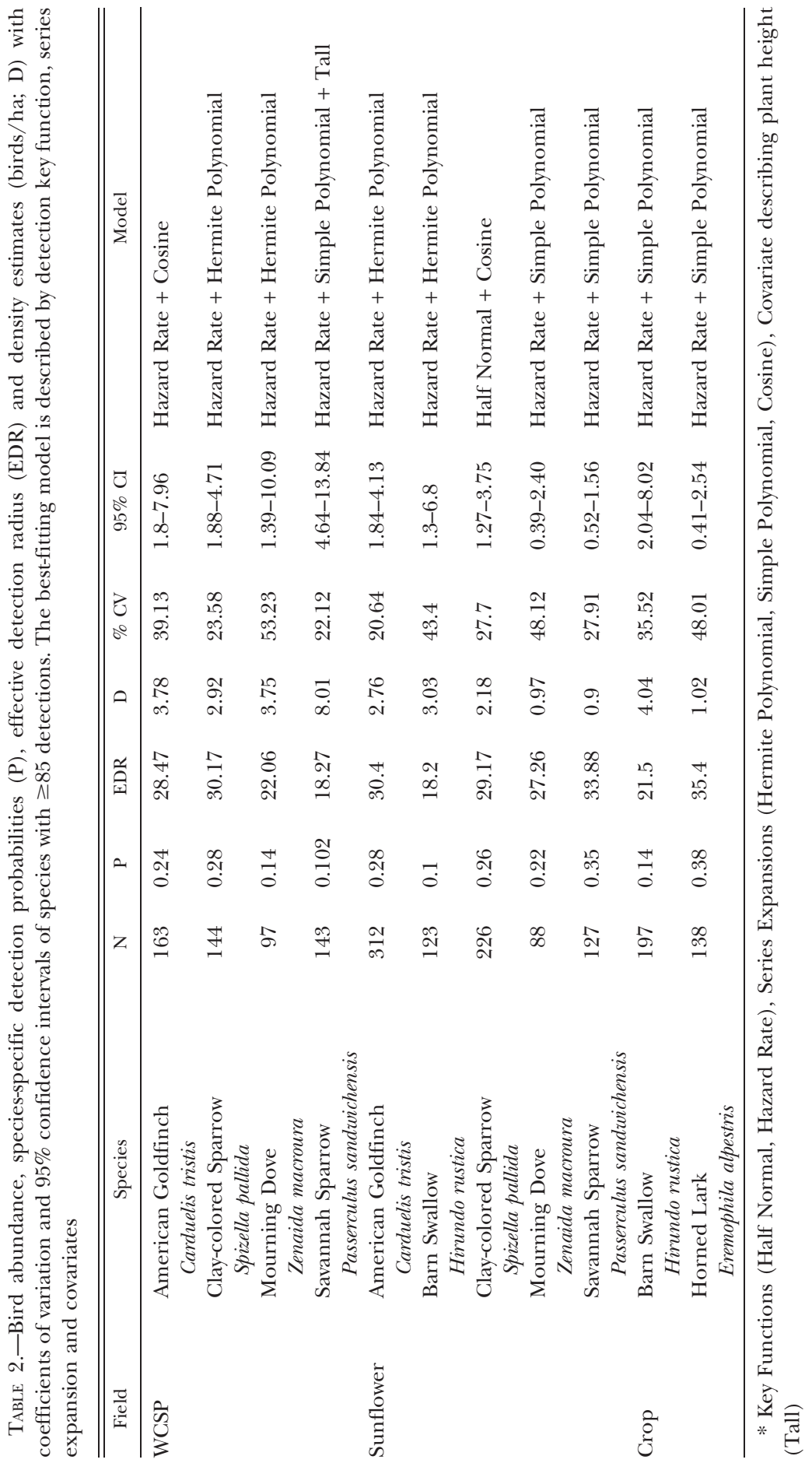


TABLE 3.-Bird 90\% confidence set with formulation technique [post hoc or a priori and intrinsic (within-field), extrinsic (surrounding land use) or mixed metrics], log-likelihood (£), and $\Delta \mathrm{AIC}_{\mathrm{c}} \mathrm{values}$, model weight $\left(w_{i}\right)$ and evidence ratios (ER)

\begin{tabular}{|c|c|c|c|c|c|}
\hline Model & Variables & $£$ & $\Delta \mathrm{AIC}_{\mathrm{c}}$ & $w_{i}$ & ER \\
\hline post hoc-intrinsic & Brassicaceae, crop density, crop height & 206.7 & 0.0 & 0.26 & 1.00 \\
\hline post hoc-mixed & $\begin{array}{l}\text { Brassicaceae, Chenopodiaceae, crop } \\
\text { density, crop height, Polygonaceae }\end{array}$ & 202.7 & 1.2 & 0.15 & 1.82 \\
\hline post hoc-mixed & $\begin{array}{l}\text { Brassicaceae, Chenopodiaceae, crop } \\
\text { density, crop height }\end{array}$ & 205.8 & 1.7 & 0.11 & 2.34 \\
\hline post hoc-mixed & Brassicaceae, crop density, wetlands & 208.5 & 1.8 & 0.11 & 2.46 \\
\hline post hoc-intrinsic & Brassicaceae, crop density & 211.0 & 1.9 & 0.10 & 2.59 \\
\hline post hoc-intrinsic & Brassicaceae, Chenopodiaceae, crop density & 209.9 & 3.2 & 0.05 & 4.95 \\
\hline post hoc-intrinsic & crop density, crop height & 212.9 & 3.8 & 0.04 & 6.69 \\
\hline post hoc-intrinsic & $\begin{array}{l}\text { Brassicaceae, Chenopodiaceae, } \\
\text { crop density, crop height, weeds }\end{array}$ & 206.6 & 4.1 & 0.03 & 7.77 \\
\hline post hoc-mixed & crop density, crop height, sunflowers & 211.6 & 5.0 & 0.02 & 12.18 \\
\hline post hoc-mixed & crop density, sunflowers & 214.6 & 5.5 & 0.02 & 15.64 \\
\hline post hoc-mixed & crop density, grasslands & 214.9 & 5.7 & 0.02 & 17.29 \\
\hline post hoc-mixed & crop density, crop height, grasslands & 212.4 & 5.7 & 0.02 & 17.29 \\
\hline
\end{tabular}

* Brassicaceae-abundance of plants from this family, Chenopodiaceae-abundance of plants from this family, Polygonaceae-abundance of plants from this family, crop density-mean crop plants $/ \mathrm{m}^{2}$, crop height-mean maximum crop height, sunflowers-area of surrounding sunflower, grasslandsarea of surrounding grass, weeds-abundance of non-crop plants, wetlands-area of surrounding wetlands

Total non-crop seed weight was similar $(\mathrm{P}=0.46)$ among WCSP $($ mean $=3.2 \mathrm{~kg} / \mathrm{ha}, \mathrm{SE}=$ $0.8)$, commercial sunflower (mean $=3.6 \mathrm{~kg} / \mathrm{ha}, \mathrm{sE}=0.9$ ) and other non-sunflower rowcrops $($ mean $=2.1 \mathrm{~kg} / \mathrm{ha}, \mathrm{SE}=0.7)$. We did not compare other vegetation metrics (i.e., row width, vertical obstruction, canopy coverage, etc.) among the three crop types as they were dependent on the crop type itself and varied considerable, especially in non-sunflower rowcrop fields [canola $(n=2)$, flax $(n=1)$, lentils $(n=2)$, soybeans $(n=18)$ and wheat $(n=$ 12)].

We evaluated 37 models constructed both a priori $(\mathrm{n}=20)$ and post hoc $(\mathrm{n}=17)$ describing our dependent variable (all bird density) and a variety of explanatory variables in WCSP. The $90 \%$ confidence set of best models (summed model weight $=0.93, \Delta \mathrm{AIC}_{\mathrm{c}}=0-$ 5.7; Table 3) included 12 models. Seven of these 12 top models included only within-field (intrinsic) habitat metrics and all 12 contained at least one intrinsic metric (Moore and Simons, 1992). The top model $\left(w_{i}=0.26\right)$ for birds in WCSP was formulated post hoc and included the intrinsic variables crop plant density (+), abundance of Brassicaceae plants (+), and maximum plant height (+; Table 3). No models consisting of only extrinsic habitat metrics (landscape variables) or models formed a priori were included in the $90 \%$ confidence set.

Crop density $\left(w_{i}=1.00\right)$ and maximum crop plant height $\left(w_{i}=0.68\right)$ were positively associated with all bird density and present in 12 and seven top models, respectively. Noncrop plants in the families Chenopodiaceae $\left[w_{i}=0.37\right.$; fireweed (Kochia spp.) and goosefoot (Chenopodium spp.) $]$ and Brassicaceae $\left[w_{i}=0.88\right.$; wild mustards (Sinapsis sp. and Brassica spp.)] were positively associated with all bird density in four and seven top models, respectively; however, Polygonaceae $\left[w_{i}=0.15\right.$; buckwheat (Polygonum spp.) ] was negatively associated with all bird density in one top model (Table 4). 
TABLE 4.-Variables included in the $90 \%$ confidence set of candidate models with the number of models entered, summed model weights $\left(\sum \omega\right)$ and model-averaged parameter estimate $(\tilde{\bar{\beta}})$ with standard error (SE)

\begin{tabular}{lcccc}
\hline \multicolumn{1}{c}{ Variable } & Models & $\sum \omega i$ & $\tilde{\beta}$ & $\mathrm{SE}$ \\
\hline Crop density & 12 & 1.000 & 0.382 & 0.903 \\
Crop height & 7 & 0.682 & 0.606 & 1.114 \\
Brassicaceae & 7 & 0.883 & 0.194 & 0.449 \\
Chenopodiaceae & 4 & 0.372 & 0.030 & 0.103 \\
Sunflowers & 2 & 0.042 & -0.002 & 0.009 \\
Grasslands & 2 & 0.033 & 0.005 & 0.015 \\
Wetlands & 1 & 0.116 & -0.022 & 0.047 \\
Weeds & 1 & 0.037 & -0.002 & 0.011 \\
Polygonaceae & 1 & 0.156 & -0.018 & 0.045 \\
\hline
\end{tabular}

\section{Discussion}

All bird and most individual species densities were greatest in WCSP compared to other sunflower and non-sunflower crop fields. Bird densities in WCSP were more strongly associated with habitat variables within-WCSP than surrounding land use; however, forb and non-crop seed abundance was similar among all field-types. We noted that in most nonsunflower crop fields, non-crop plants were small and often immature, although we did not quantify physical characteristics of non-crop plants in fields. In comparison, non-crop plants were often large and mature, flowering or with seed, in sunflower fields. This is likely due to later harvest and reduced use of herbicides in sunflower fields compared to other crops, especially soybeans. Plants of the family Brassicaceae continue to bloom in late summer and fall possible attracting insects and thus insectivores. Kochia spp. and other Chenopodiaceae plants prolifically produce seeds that are eaten by granivorous birds. Furthermore, the smaller size of WCSP, resulting in greater edge to interior ratio compared to other fields, may have also contributed to higher bird densities (Yahner, 1988).

Previous studies have shown that at least 94 bird species use sunflower and other crop fields during the fall and spring in the NGP (Schaaf, 2003; Galle, 2005; Hagy et al., 2007). Hagy et al. (2007) reported the occurrence of 12 species of conservation concern in the PPR using sunflower during migration (USDI, 2002; Rich et al., 2004; Butcher et al., 2007). We observed one species of conservation concern (USDI, 2002; Rich et al., 2004; Butcher et al., 2007) in WCSP (bobolink, Dolichonyx oryzivorus, $\mathrm{n}=16$ ), two in commercial sunflower (bobolink, $\mathrm{n}=31$; northern harrier, Circus cyaneus, $\mathrm{n}=1$ ) and two in non-sunflower crop fields (Franklin's gull, Larus pipixcan, $\mathrm{n}=1$; lark sparrow, Chondestes grammacus, $\mathrm{n}=10$ ) during point counts. Compared to other row crops, sunflower was more commonly used by migratory birds in the fall and spring in the NGP (Galle, 2005; Hagy et al., 2007).

Surprisingly few studies have examined bird use of active agricultural fields, given widespread cultivation of lands in North America. Best et al. (2001) examined the influence of landscape composition on bird use in row-crop fields, but did not compare that with intrinsic characteristics of the crop fields themselves. Lokemoen and Beiser (1997) found that bird density was greatest in the fall in conventional cropland fields compared to minimum tillage and organic-management crop fields. We have noted recent works that excluded agriculture lands from habitat analyses (Ribic et al., 2009a) even though agriculture dominated the study area and birds used those habitats (Best et al., 2001; Hagy et al., 2007), and others that refer to agricultural lands as "hostile" (Ribic et al., 2009b). 
While agricultural lands may exhibit considerable temporal structural variability compared to grassland habitats, we note the variety of species that use them during fall in North Dakota. Furthermore, we observed greater bird densities in sunflower and especially in WCSP than previously reported during fall in fallow, sunflower and wheat fields (Lokemoen and Beiser, 1997); in cornfields during the breeding season (Best et al., 1990); in Great Plains forest fragments in spring (Martin, 1980); and in Conservation Reserve Program grasslands and row-crop fields in winter (Best et al., 1998).

Birds were more closely associated with vegetation characteristics within WCSP than landuse outside of the lure plots. Seven of the 12 top models contained only within-field habitat metrics and the top four model averaged scores were from within-field metrics. Contrary to results describing response of blackbirds to surrounding landscape variables reported by Hagy et al. (2008) and Cummings et al. (1987), non- blackbird avian species may select crop fields using proximate cues within-fields rather than landscape composition at the $2.4 \mathrm{~km}$ scale in our study area. In east-central North Dakota, abundant wetlands, grasslands and agricultural fields may facilitate a selection response to the region (in this case, our $\sim 60,000 \mathrm{~km}^{2}$ study area) rather than small subunits of the landscape, such as our $2.4 \mathrm{~km}$ site area. It is possible that birds in this study selected the landscape at a scale larger than $2.4 \mathrm{~km}$, but due to logistical and temporal restraints we were not able to test this hypothesis.

We determined that tall $(\geq 1.3 \mathrm{~m})$, densely planted within rows $(\geq 5$ crop plants / linear m), WCSP with some non-crop plants (forbs within Brassicaceae and Chenopodiaceae families) resulted in the greatest bird densities in WCSP. Although landscape variables received less model weight, ha of surrounding grasslands were positively associated while ha of sunflowers and wetlands were negatively associated with all bird density in WCSP. Commercial sunflower and wetlands with emergent vegetation likely provide alternative habitat for migrating birds, which may explain a negative correlation with bird densities in WCSP. Additionally, blackbirds greatly reduced sunflower seed abundance in some WCSP near wetlands, which may have reduced food resources for other granivorous birds (Hagy et al., 2008). We recommend planting WCSP with dense plant spacing within rows, and using management practices that allow non-crop plants to persist. Wide row spacing and initial site preparation with little subsequent between-row tillage will likely result in tall, vegetatively diverse WCSP attractive to a variety of bird species. Furthermore, planting WCSP near cattail-dominated wetlands and shelterbelts that blackbirds use as roosting and loafing sites may increase effectiveness as a pestmanagement system (Hagy et al., 2008).

Our results are consistent with the findings of Cunningham and Johnson (2006) that landscape variables alone were not as closely associated with bird distributions as either models consisting of only within-field (intrinsic proximate cues) or within-field and landscape-level variables. These authors also reported closer bird-landscape associations at large scales $(800-1600 \mathrm{~m})$ for most grassland species they encountered; however, at the $2.4 \mathrm{~km}$ scale, we did not detect similar associations. Similarly, Ribic et al. (2009a) found that grassland birds were more strongly associated with habitat type than surrounding landscape metrics. We suggest further research be conducted at large $(>2.4 \mathrm{~km})$ or multiple spatial and temporal scales in agricultural fields during non-breeding periods to examine both intrinsic and extrinsic habitat variables in comparison to bird densities.

Hagy et al. (2008) reported the cost of planting 41 WCSP in 2004 and 2005 for blackbird damage prevention to exceed direct savings from damage amelioration by a factor of 2.3:1. However, individual producers were not subject to a deficit because of the cost-share 
program. Furthermore, benefits of migratory bird and wildlife habitat could further offset costs substantially to sunflower producers. Cost-share planting, land-lease for hunting, and difficult to quantify factors such as migratory bird habitat likely increase the value of WCSP to producers.

It is difficult to quantify non-market values of wildlife habitat, especially for non-game animals (Boyer and Polasky, 2004). However, we approximated the value of wildlife habitat of WCSP using economic incentives offered by the Private Land Initiative Food Plot Program for sportsman implemented by the North Dakota Game and Fish Department. The food plot program awards as much as $\$ \mathrm{US} 46 /$ ha to private landowners for planting rowcrop food plots for wildlife and allowing walk-in access. Combining these economic incentives, as a surrogate valuation of wildlife food plots, with blackbird damage reduction would result in a 1.8:1 cost (subsidization $\$ \mathrm{US} 375 / \mathrm{ha}$ ) to benefits (average value of damage reduction provided by plots + value of food plots for wildlife) ratio on average. To implement the program at a 1:1 cost-benefit ratio and make subsidization economically sustainable for Wildlife Services, other ecological services and non-market value of WCSP would need to total \$US195/ha.

Wildlife Services subsidized sunflower producers $\$$ US375/ha to plant WCSP in 20042005. A highly competitive applicant pool of sunflower producers indicated that participation in the program was economically beneficial for producers at that rate. If producers were willing to participate in the program for reduced compensation, then it is likely that the benefits might further outweigh the costs associated with the WCSP program. Additionally, these cost-benefit analyses was applied using all of the plots in our study area and mean blackbird damage estimates from Hagy et al. (2008). Some individual WCSP were economically viable considering damage amelioration alone because they were depredated upwards of $80 \%$ by blackbirds, yet still provided fall-winter habitat and foods for wildlife. Using placement and management recommendations from this study and Hagy et al. (2008), WCSP can be maximized to provide an economically viable wildlife habitat/nonlethal blackbird damage control system. We suggest further implementation of the WCSP program using our management recommendations and further evaluation including economic quantification of benefits provided by WCSP.

WCSP can accommodate a wide variety of migratory birds while providing a row crop/ blackbird damage control system for farmers in the northern Great Plains. Integrative pest management/wildlife management systems may become increasingly important in the NGP as sunflower acreages have declined by almost $50 \%$ while corn $(+250 \%)$ and soybeans $(+100 \%)$, both with glyphosate tolerant varieties, have become increasingly common in North Dakota since 1998 (USDA, 2008). Conversion of sunflower and grassland habitats in the NGP to other habitats could negatively affect migratory birds given their abundance in these habitats compared to other croplands (Otis and Kilburn, 1988). Furthermore, decreasing sunflower could concentrate blackbirds and require more intensive and lethal pest management techniques, which may negatively affect other wildlife. If economically viable, continued sunflower production and WCSP implementation may benefit migratory birds by providing better habitat than other row-crops in the NGP.

Acknowledgments. - We thank the United States Department of Agriculture Wildlife Service's National Wildlife Research Center, North Dakota Wildlife Services and North Dakota State University Department of Biological Sciences for funding and support. We thank NDSU graduate students and field and laboratory assistants for their support and dedicated service. We thank S. K. Riffell for suggestions pertaining to our statistical analysis and R. M. Bush, G. M. Forcey, E. H. Hagy, M. McConnell and M. L. Schummer for evaluating early versions of this manuscript. 


\section{Literature Cited}

Akaike, H. 1974. A new look at the statistical model identification. IEEE Trans. Auto. Contr., 19:716-723. Alldredge, M. W., K. H. Pollock, T. R. Simons and S. A. Shriner. 2007. Multiple-species analysis of point count data: a more parsimonious modeling framework. J. Appl. Ecol., 44:281-290.

Anderson, D. R., K. P. Burnham and W. L. Thompson. 2000. Null hypothesis testing: problems, prevalence, and an alternative. J. Wildl. Manage., 64:912-923.

Bergin, T. M. 1992. Habitat selection by the western kingbird in western Nebraska: a hierarchical analysis. Condor, 94:903-911.

Best, L. B., T. M. Bergin and K. E. Freemark. 2001. Influence of landscape composition on bird use of rowcrop fields. J. Wildl. Manage., 65:442-449.

, R. C. Whitmore and G. M. Booth. 1990. Use of corn fields by birds during the breeding season: the importance of edge habitat. Am. Midl. Nat., 123:84-99.

— , H. Campa, K. E. Kemp, R. J. Robel, M. R. Ryan, J. A. Savidge, H. P. Weeks, Jr. and S. R. Winterstein. 1998. Avian abundance in CRP and crop fields during winter in the midwest. Am. Midl. Nat., 139:311-324.

Boyer, T. AND S. POLASKy. 2004. Valuing urban wetlands: a review of non-market valuation studies. Wetlands, 124:744-755.

Buckland, S. T., D. R. Anderson, K. P. Burnham, J. L. Locke, D. L. Borchers and L. Thomas. 2001. Introduction to distance sampling: estimating abundance of biological populations. Oxford University Press, Inc., New York, New York. 432 p.

Burnham, K. P. and D. R. Anderson. 2002. Model selection and multimodel inference. Spring Verlag, Inc., New York, New York. 488 p.

Butcher, G. S., D. K. Niven, A. O. Panjabi, D. N. Pashley and K. V. Rosenburg. 2007. The 2007 watchlist for United States birds. Am. Birds, 61:18-25.

Charlet, L. D., G. J. Brewer and B. A. Franzman. 1997. Sunflower insects, p. 183-261. In: A. A. Schneiter (ed.). Sunflower, Technology, and Production. Agronomy Monograph, No. 35. American Society of Agronomy, Crop Science Society of America, and Soil Science Society of America, Madison, Wisconsin.

Crozier, G. E. and G. J. Niemi. 2003. Using patch and landscape variables to model abundance in a naturally heterogeneous landscape. Can. J. Zool., 81:441-452.

Cummings, J. L., J. L. Guarino, C. E. Knittle and W. C. Royal, JR. 1987. Decoy plantings for reducing blackbird damage to nearby commercial sunflower fields. Crop Prot., 6:56-60.

Cunningham, M. A. and D. A. Johnson. 2006. Proximate and landscape factors influence grassland bird distributions. Ecol. Appli., 16:1062-10-75.

EfFord, M. G. AND D. K. Dawson. 2009. Effect of distance-related heterogeneity on population size estimates from point counts. Auk, 126:100-111.

Environmental Systems Research Institute, Inc. (ESRI). 2004. ArcMap version 9.1. Redlands, California.

Flather, C. H. and J. R. Sauer. 1996. Using Landscape Ecology to test Hypotheses about large-scale abundance patterns in migratory birds. Ecology, 77:28-35.

Galle, A. G. 2005. Avian use of harvested crop fields during spring migration through the southern drift plains regions of North Dakota. M.S. thesis. North Dakota State University, Fargo. 146 p.

Hagy, H. M., G. M. Linz and W. J. Bleier. 2007. Are sunflower fields for the birds? p. 61-71. In: D. L. Nolte, W. M. Arjo and D. L. Stalman (eds.). Proceedings of the Twelfth Wildlife Damage Management Conference. Corpus Christie, Texas.

- - - AND 2008. Optimizing decoy crops for blackbird control in commercial sunflower. Crop Prot., 27:1442-1447.

Johnson, D. H. 1980. The comparison of usage and availability measurements for evaluating resource preference. Ecology, 61:65-71.

Johnson, J. B. and K. S. Omland. 2004. Model selection in ecology and evolution. Trends Ecol. Evol., 19:101-108.

Kleingartner, L. 2003. Sunflower losses to blackbirds: an economic burden, p. 13-14. In: G. M. Linz (ed.). Management of North American blackbirds. National Wildlife Research Center, Fort Collins, Colorado, USA. 
Koford, R. R. AND L. B. Best. 1996. Management of agricultural landscapes for the conservation of neotropical migratory birds, p. 68-88. In: F. Thompson (ed.). Management of midwestern landscapes for the conservation of neotropical migratory birds. USDA, Forest Service General Technical Report, NC-187. North Central Forest Experiment Station, St. Paul, Minnesota.

Kolasa, J. 1989. Ecological systems in hierarchical perspective: breaks in community structure and other consequences. Ecology, 70:36-47.

Kotliar, N. B. And J. A. Wiens. 1990. Multiple scales of patchiness and patch structure: a hierarchical framework for the study of heterogeneity. Oikos, 59:253-260.

Linz, G. M., H. J. Homan, L. B. Penry and P. Mastrangelo. 2003. Reducing blackbird-human conflicts in agriculture and feedlots: new methods for an integrated management approach, p. 21-24. In: G. M. Linz (ed.). Management of North American blackbirds. National Wildlife Research Center, Fort Collins, Colorado.

D. A. Schaff, P. Mastrangelo, H. J. Homan, L. B. Perry and W. J. Bleier. 2004. Wildlife conservation sunflower plots as a dual purpose wildlife management strategy, p. 291-294. In: R. M. Timm and W. B. Gorenzel (eds.). Proceedings of the 21st Vertebrate Pest Conference. Visalia, California.

Littell, R. C., G. A. Milliken, W. W. Stroup, R. D. Wolfinger and O. Schabenberger. 1996. SAS system for mixed models. SAS Institute, Cary, North Carolina. 814 p.

Lokemoen, J. T. And J. A. Beiser. 1997. Bird use and nesting in conventional, minimum tillage, and organic cropland. J. Wildl. Manage., 61:644-655.

Lubowski, B. N., M. Vesterby, S. Bucholtz, A. Baez and M. J. Roberts. 2006. U. S. Department of Agriculture Economics Research Service - major uses of land in the United States, 2002. EIB. 14. 54 p. http://www.ers.usda.gov/Data/MajorLandUses/MLUsummary tables.pdf.

Marques, T. A., L. Thomas, S. G. Fancy and S. T. Buckland. 2007. Improving estimates of bird density using multiple-covariate distance sampling. Auk, 124:1229-1243.

Martin, T. E. 1980. Diversity and abundance of spring migratory birds using habitat islands on the Great Plains. Condor, 82:430-439.

McCune, B. And J. B. Grace. 2002. Analysis of ecological communities. MjM Software Design, Gleneden Beach, Oregon. 300 p.

Moore, F. R., S. A. Gauthreaux, Jr., P. Kerlinger and T. R. Simons. 1995. Habitat requirements during migration: important link in conservation, p. 121-144. In: T. Martin and D. M. Finch (eds.). Ecology and management of neotropical migratory birds. Oxford University Press, New York, New York.

And D. A. Aborn. 2000. Mechanisms of en route habitat selection: how do migrants make habitat decisions during stopover. Studies Avian Bio., 20:34-42.

AND T. R. Simons. 1992. Habitat suitability and stopover ecology of neotropical landbird migrants, p. 345-355. In: J. M. Hagan and D. W. Johnson (eds.). Ecology and conservation of neotropical migrant landbirds. Smithsonian Institution Press, Washington D.C.

Murphy, M. T. 2003. Avian population trends within the evolving agricultural landscape of eastern and central United States. Auk, 120:20-34.

Otis, D. L. And C. M. Kilburn. 1988. Influence of environmental factors on blackbird damage to sunflower. Fish and Wildlife Service Technical Report 16. U.S. Department of the Interior, Fish and Wildlife Service, Bethesda, Maryland. 11 p.

Pearse, A. T. 2007. Design, evaluation, and applications of an aerial survey to estimate abundance of wintering waterfowl in Mississippi. Dissertation, Mississippi State University, Mississippi State, USA. $170 \mathrm{p}$.

Peer, B. D., H. J. Homan, G. M. Linz and W. J. Bleier. 2003. Impact of Blackbird damage to sunflower: bioenergetic and economic models. Ecol. Appl., 13:248-256.

Peterjohn, B. P. 2003. Agricultural landscapes: can they support healthy bird populations as well as farm products? Auk, 120:14-19.

Ribic, C. A., M. J. Guzy And D. W. SAmple. 2009a. Grassland bird use of remnant prairie and conservation reserve program fields in an agricultural landscape in Wisconsin. Am. Midl. Nat., 161:110-122. 
, R. R. Koford, J. R. Herkert, D. H. Johnson, N. D. Niemith, D. E. Naugle, K. K. Bakker, D. W. Sample and R. B. Renfrew. 2009b. Area sensitivity in North American grassland birds: patterns and processes. Auk, 126:233-244.

Rich, T. D., C. J. Beardmore, H. Berlanga, P. J. Blancher, M. S. W. Bradstreet, G. S. Butcher, D. W. Demarest, E. H. Dunn, W. C. Hunter, E. E. Iñigo-Elias, J. A. Kennedy, A. M. Martell, A. O. Panjabi, D. N. Pashley, K. V. Rosenberg, C. M. Rustay, J. S. Wendt and T. C. Will. 2004. Partners in flight North American landbird conservation plan. Cornell Laboratory of Ornithology, Ithaca, New York. http://www.partnersinflight.org/cont_plan/PIF4_AppendicesWEB.pdf

Riffell, S. K., T. Burton And M. Murphy. 2006. Birds in depressional forested wetlands: area and habitat requirements and model uncertainty. Wetlands, 26:107-118.

SAS Institute Inc., SAS 9.1.3. 2000-2005. The mixed procedure, Cary, North Carolina, USA.

Sauer, J. R., J. E. Hines and J. Fallon. 2008. The North American Breeding Bird Survey, Results and Analysis 1966-2007. Version 5.15.2008. USGS Patuxent Wildlife Research Center, Laurel, Maryland, USA.

SchaAf, D. A. 2003. Avian use of ripening sunflower fields. M.S. Thesis, North Dakota State University, Fargo, North Dakota. 113 p.

Stewart, R. E. 1975. Breeding birds of North Dakota. Tri-college center for environmental studies. Fargo, North Dakota. 295 p.

Stone, C. P. And C. R. Danner. 1980. Autumn flocking of red-winged blackbirds in relation to agricultural variables. Am. Midl. Nat., 130:196-199.

Thomas, J. L., S. Strindberg, F. F. C. Marques, S. T. Buckland, D. L. Borchers, D. R. Anderson, K. P. Burnham, S. L. Hedley, J. H. Pollard, J. R. B. Bishop and T. A. Marques. 2005. DistancE version 5.0 beta 5. Research Unit for Wildlife Population Assessment, University of St. Andrews, United Kingdom.

U. S. Department of Agriculture [USDA]. 2008. Statistics by state - Quick stats. National Agricultural Statistics Service, Fairfax, Virginia. http://www.nass.usda.gov/Statistics_by_State/North_ Dakota/index.asp.

U.S. Department of Agriculture National Resources Conservation Service [USDA]. 2005. Geospatial data gateway. http://datagateway.nrcs. usda.gov.

U. S. Department of Interior [USDI]. 2002. Birds of conservation concern 2002. USDI, Fish and Wildlife Service, Division of Migratory Bird Management, Arlington, Virginia. http://www.migratorybirds. fws.gov/reports/bcc2002.pdf.

Uyehara, K. J., A. Engilis, Jr. And B. D. Dugger. 2008. Wetland features that influence occupancy by the endangered Hawaiian duck. Wilson J. Ornith., 120:311-319.

Whittaker, R. H. 1972. Evolution and measurement of species diversity. Taxon, 21:213-251.

YAHner, R. H. 1988. Changes in wildlife communities near edges. Conserv. Biol., 2:333-339.

ZaR, J. H. 1999. Biostatistical analysis, 3rd ed. Prentice Hall, Englewood Cliffs, New Jersey. 944 p. 